\title{
POSITRONIUM AS A PROBE FOR NEW PHYSICS BEYOND THE STANDARD MODEL ${ }^{1}$
}

\author{
A. Rubbia ${ }^{2}$ \\ Institut für Teilchenphysik, ETHZ, CH-8093 Zürich, Switzerland
}

\begin{abstract}
We discuss and summarize some aspects concerning the study of the positronium system to probe new physics beyond the Standard Model.
\end{abstract}

Keywords: Positronium, new physics, extra-dimension, mirror matter, dark matter

\footnotetext{
${ }^{1}$ Talk given at the Workshop on Positronium Physics, Zurich (Switzerland), 30-31 May 2003.

22Andre.Rubbia@cern.ch
} 


\section{Introduction}

Quantum electrodynamics (QED) is the textbook example of the success of quantum field theory. Many physical quantities (anomalous electron and muon magnetic moments, hyperfine splitting of hydrogen, muonium and positronium, Lamb shift, etc.) have been calculated very precisely. The measurements have been characterized by excellent agreement with the theoretical predictions and in general provided very clean conditions for hunting for small deviations from the standard theory.

Positronium $(P s)$, the positron-electron bound state, is the lightest known atom, which is bounded and self-annihilates through the same, electromagnetic interaction. At the current level of experimental and theoretical precision this is the only interaction present in this system. This feature has made positronium an ideal system for testing the accuracy of QED calculations for bound states, in particular for the triplet $\left(1^{3} S_{1}\right)$ state of $P s$, orthopositronium $(o-P s)$. Due to the odd-parity under C-transformation $o-P s$ decays predominantly into three photons. As compared with the singlet $\left(1^{1} S_{0}\right)$ state (parapositronium), the "slowness" of $o-P s$ decay rate, due to the phase-space and additional $\alpha$ suppression factors, gives an enhancement factor $\simeq 10^{3}$, making it more sensitive to an admixture of new interactions which are not accommodated in the Standard Model.

Positronium was discovered experimentally by Deutsch 1 in 1951, who observed its decay in different gases. Since then, a lot of focus has been set on the determination of its basic properties like decay life time, decay modes, spectroscopy, etc. In particular, the measurement of the o-Ps lifetime caught much attention.

Since the 1989, the precision on the o-Ps lifetime reached a value well under $1000 \mathrm{ppm}$. Much excitement arose when the measurements performed by the Michigan group did not agree with theory. This problem, called the o-Ps-lifetime-puzzle, ignited much experimental and theoretical activity devoted to its clarification. These are: (1) new direct lifetime measurements by the Tokyo group which did not confirm the discrepancy (2) new theoretical calculations by Adkins et al. including higher order terms improving the theoretical precision well below experimental errors, however, confirming early theoretical estimates (3) searches for "exotic" decay modes which could explain the lifetime discrepancy at the cost of new physics (violation of basic conservation laws with decays into 1 photon, 2 photons; anomalous rate in 5 photons; millicharged particles; new bosons, ...) (4) exotic suggestions for disappearance mechanisms (mirror worlds, extra dimensions). So far none of these provided a clear solution to the o-Ps-lifetimepuzzle.

Just before the Positronium physics workshop held in Zurich in May 2003, the Michigan group has published a new result which is now in agreement with the theoretical value, somewhat in contradiction with the earlier results from the same group. The easy shortcut is to assume that the o-Ps-lifetime puzzle is closed. Should we however indeed consider that the o-Ps lifetime puzzle is solved and hence assume that further precise measurements of positronium (o-Ps lifetime, ...) are irrelevant?

During the workshop we tried to obtain a modern view on this problem, in particular in the context of the physics of positronium. More generally and in addition to the puzzle of the orthopositronium lifetime, we tried to address the study of positronium as a probe for new physics beyond the Standard Model. In this paper, we review some of these aspects.

In particular, we discuss the ideas for an "appearance" of an effect, namely through the search for the invisible decay of the o-Ps, i.e. a photon-less decay. It may be worthwhile to remember that the process with analogous experimental signature, $Z \rightarrow$ invisible decay plays a fundamental role in the determination of the number of lepton families. This is to be contrasted with the "disappearance" searches, where one measures the o-Ps-lifetime as precisely as possible and then looks for a deviation of this result from the theoretical one. In the disappearance mode, one is looking for a small effect. In the appearance mode, we are looking for few events with energy deposition in our detector compatible with zero, a direct experimental signature that cannot happen if o-Ps decays to standard particles. New experiments are being designed (see Section [6) to reach a sensitivity in the branching ratio at the level 
of $10^{-8}$.

In addition, we mention the fact that a new experiment capable for investigating the invisible o-Ps decay down to $10^{-8}$ could test the existence of extra-dimensions. This could turn out to be a new domain of research and stresses once again the known fact that precise experiments at low energy can compete with big projects at the highest energy frontiers. Note that within the Standard electroweak model, orthopositronium can decay invisibly into a neutrino-antineutrino pair. The $o-P s \rightarrow \nu_{e} \bar{\nu}_{e}$ decay occurs through $W$ exchange in the $t$-channel and $e^{+} e^{-}$annihilation via $Z$. The decay width is 44]

$$
\Gamma\left(o-P s \rightarrow \nu_{e} \bar{\nu}_{e}\right) \approx 6.2 \cdot 10^{-18} \Gamma_{3 \gamma}
$$

For other neutrino flavours only the $Z$-diagram contributes. For $l \neq e$ the decay width is [44]

$$
\Gamma\left(o-P s \rightarrow \nu_{l} \bar{\nu}_{l}\right) \approx 9.5 \cdot 10^{-21} \Gamma_{3 \gamma}
$$

Thus, in the SM the $o-P s \rightarrow \nu \bar{\nu}$ decay rate is very small and evidence for invisible decays would unambiguously signal the presence of new physics.

Overall, precise study of positronium could yield new information on fundamental discrete symmetries like charge conjugation $(\mathrm{C})$, parity $(\mathrm{P})$, time-reversal $(\mathrm{T}), \mathrm{CP}$ and even $\mathrm{CPT}$, continuing the long tradition of tests of these fundamental symmetries in atomic systems.

Of course, the study of positronium cannot be accomplished without a precise theoretical understanding of the QED predictions. We also collected for the workshop theoretical contributions in the context of precision tests of the QED theory, high order QED corrections, connection between positronium and quarkonium.

During this workshop we also wanted to have a critical review of the existing o-Ps-lifetime results and planned some talks accordingly, which resulted in interesting discussions during the meeting.

Finally, one aspect that has been neglected in this introduction up to now is the possible application of the experimental techniques developed in the context of fundamental positronium physics to the applied science of materials. To address this point, we have invited two talks related to the use of positron annihilation for the characterization of solids and polymers.

\section{Renaissance of the mirror world?}

In 1956 Lee and Yang proposed 2 that the weak interactions of fundamental particles were not invariant under the parity transformation. They suggested it to explain some experimental results that were considered as puzzles. They pointed out that these puzzles could be explained if one assumed a left-right asymmetry in the weak interactions. In their original paper, they also provided a "remedy" for this a priori not sensible assumption. Indeed Lee and Yang argued 2] that if left-right asymmetry were found in weak interactions, the question could still be raised whether there could not exist corresponding elementary particles exhibiting opposite asymmetry such that in the broader sense there will still be over-all right-left symmetry. They spoke specifically of two kinds of protons, the left-handed and the right-handed one. As well known, left-handed nature of ordinary matter was brilliantly confirmed experimentally. Nowadays parity violation in fundamental interactions is so well accepted, that the left-right asymmetry of Nature is inserted in the modern Standard Model "from the beginning" in the assignment of the particle fields. Nonetheless, the question whether Nature is fundamentally left-right symmetric or not has remained up to now unresolved.

Landau was always convinced by the absolute symmetry of vacuum [3. Under his appeal Kobzarev, Okun and Pomeranchuk suggested the hypothesis of a mirror world 4 .

It is fair to say that the existence of mirror matter has been recently boosted since the realization of its possible connection to the dark matter problem. If about $30 \%$ of the critical density of the Universe is 
composed of non-baryonic dark matter, as recent astrophysical observations seem to indicate, then this clearly motivates the search for new kinds of matter, since the current Standard Model does not contain any heavy, stable non-baryonic particles.

The idea that there can exist a hidden mirror sector of particles and interactions which is the exact duplicate of our visible world has attracted a significant interest over last years and has been summarized at this workshop by Berezhiani [5]. The basic concept is to have a theory given by the product $G \times G^{\prime}$ of two identical gauge factors with the identical particle contents, which could naturally emerge e.g. in the context of $E_{8} \times E_{8}^{\prime}$ superstring.

As discussed by Foot $\underline{6}$, the ordinary and mirror particles could form parallel sectors each with gauge symmetry $G$ (where $G=G_{S M} \equiv S U(3)_{c} \otimes S U(2)_{L} \otimes U(1)_{Y}$ in the simplest case) so that the full gauge group is $G \otimes G$. Mathematically, mirror symmetry has the form: 4]

$$
\begin{gathered}
x \rightarrow-x, t \rightarrow t, \\
W^{\mu} \leftrightarrow W_{\mu}^{\prime}, B^{\mu} \leftrightarrow B_{\mu}^{\prime}, G^{\mu} \leftrightarrow G_{\mu}^{\prime} \\
\ell_{i L} \leftrightarrow \gamma_{0} \ell_{i R}^{\prime}, e_{i R} \leftrightarrow \gamma_{0} e_{i L}^{\prime}, q_{i L} \leftrightarrow \gamma_{0} q_{i R}^{\prime}, u_{i R} \leftrightarrow \gamma_{0} u_{i L}^{\prime}, d_{i R} \leftrightarrow \gamma_{0} d_{i L}^{\prime},
\end{gathered}
$$

where $G^{\mu}, W^{\mu}, B^{\mu}$ are the standard $G_{S M}$ gauge particles, $\ell_{i L}, e_{i R}, q_{i L}, u_{i R}, d_{i R}$ are the standard leptons and quarks $(i=1,2,3$ is the generation index) and the primes denote the mirror particles.

Ordinary and mirror particles couple with each other via gravity and possibly by new interactions connecting ordinary and mirror particles together. Constraints from gauge invariance, mirror symmetry and renormalizability, suggest only two types of new interactions [4: a) Higgs-mirror Higgs quartic coupling $\left(\mathcal{L}=\lambda^{\prime} \phi^{\prime \dagger} \phi^{\prime} \phi^{\dagger} \phi\right)$, and b) via photon-mirror photon kinetic mixing:

$$
\mathcal{L}_{i n t}=\frac{\epsilon}{2} F^{\mu \nu} F_{\mu \nu}^{\prime}
$$

where $F^{\mu \nu}\left(F_{\mu \nu}^{\prime}\right)$ is the field strength tensor for electromagnetism (mirror electromagnetism). The effect of photon-mirror photon kinetic mixing is to cause mirror charged particles to couple to ordinary photons with effective electric charge $\epsilon e$ [4, 7, 8].

\subsection{Mirror world and invisible decays of positronium}

Glashow pointed out 9 that a sensitive laboratory test for mirror matter comes from the orthopositronium system. The interaction of $e^{+} e^{-}$with $e^{\prime} e^{\prime}-$ leads to a small mass term mixing orthopositronium with mirror orthopositronium. The effect of this mass mixing term is to cause orthopositronium to (maximally) oscillate into mirror orthopositronium [10]:

$$
P\left(o-P s \rightarrow o-P s^{\prime}\right)=\sin ^{2} \omega t,
$$

where $\omega=2 \pi \epsilon f$, where $f=8.7 \times 10^{4} \mathrm{MHz}$ is the contribution to the ortho-para splitting from the one photon annihilation diagram involving orthopositronium (see Figure 1).

In an experiment, mirror orthopositronium decays are not detected, hence leading to $o-P s \rightarrow$ invisible decays, which means that the number of orthopositronium, $N$, satisfies 9

$$
N=\cos ^{2} \omega t e^{-\Gamma^{3 \gamma} t} \approx \exp \left[-t\left(\Gamma^{3 \gamma}+\omega^{2} t\right)\right]
$$

where $\Gamma^{3 \gamma}$ is the standard orthopositronium decay rate. Evidently, the observational effect of the oscillations is to increase the apparent decay rate of ordinary orthopositronium: $\Gamma^{e f f} \approx \Gamma^{3 \gamma}\left(1+\omega^{2} / \Gamma^{3 \gamma}\right)$. This implies that the existence of mirror matter can be probed with positronium either (1) via a precision measurement of its decay rate and/or shape of its decay time spectrum or (2) via a direct search for invisible decays. 


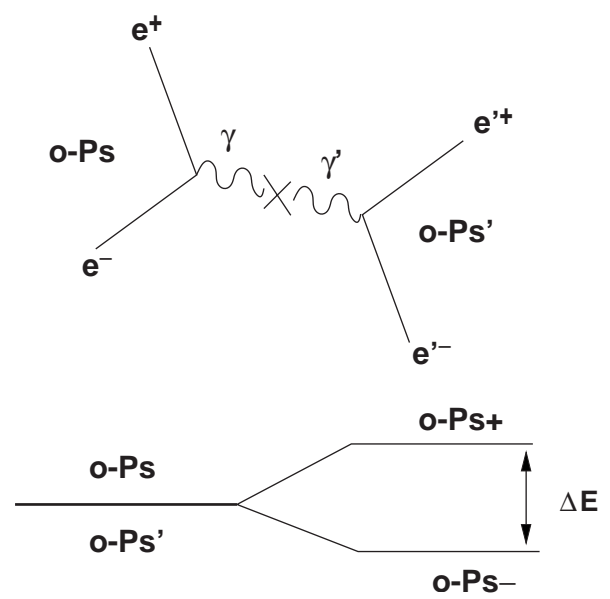

Figure 1: The double degeneracy between orthopositronium mass eigenstates of ordinary o-Ps and mirror o-Ps' is broken when a small mixing (upper picture) term is included.

In the simplest case of $o-P s \rightarrow o-P s^{\prime}$ oscillations in vacuum [9] the branching ratio occurring during a long enough observation time can be calculated as

$$
B r(o-P s \rightarrow \text { invisible })=\frac{2(2 \pi \epsilon f)^{2}}{\Gamma_{3 \gamma}^{2}+4(2 \pi \epsilon f)^{2}}
$$

However, Eq.(7) may not be applicable to all measurements. In experiments, orthopositronium is not produced in vacuum, but rather by slow positron collisions with a positronium formation target. As a result, the newly formed positronium will undergo elastic collisions with the target at a rate, $\Gamma_{\text {coll }}$, which depends on the particular experiment. These collisions cause quantum decoherence, disrupting the ordinary-mirror oscillations.

Hence, it is absolutely fundamental to distinguish experiments in "vacuum", where o-Ps is contained for example in a large evacuated cavity, from other setups where o-Ps undergoes numerous interactions with the environment within its lifetime. This situation is summarized in Table 1 where the three types of experiments (gas 11, vacuum cavity 12 and powder 13, see Section 44) are illustrated. It is well known that collisions damp the oscillations, e.g. in the limit where the collision rate is much larger than the decay rate (or oscillation frequency, whichever is smaller) the effect of the oscillations becomes negligible. In addition, external fields might result in a loss of coherence due to additional splitting of mass eigenstates.

Table 1: Some measurements of the orhopositronium lifetime. The last column is an estimate of the mean collision rate in the experiment (from Ref.[10]).

\begin{tabular}{ccccc}
\hline group/ref & Rate $\Gamma_{3 \gamma}$ & $\begin{array}{c}\text { errors } \\
\mu s^{-1}\end{array}$ & Technique & $\Gamma_{\text {coll }}$ \\
\hline Ann Arbor [11] $]$ & 7.0514 & 200 & Gas & $\approx 10^{3} \Gamma_{3 \gamma}$ \\
Ann Arbor[12] & 7.0482 & 230 & Vacuum & $\approx(3-10) \Gamma_{3 \gamma}$ \\
Tokyo [13]] & 7.0398 & 412 & Powder & $\approx 10^{4} \Gamma_{3 \gamma}$
\end{tabular}

Let us consider the case where the collision rate is much larger than the decay rate, $\Gamma_{\text {coll }} \gg \Gamma_{3 \gamma}$, then the observed decay rate is approximately given by [10]:

$$
\Gamma_{\text {obs }} \simeq \Gamma_{3 \gamma}+\frac{2 \omega^{2}}{\Gamma_{\text {coll }}}=\Gamma_{3 \gamma}\left(1+\frac{2(2 \pi \epsilon f)^{2}}{\Gamma_{\text {coll }} \Gamma_{3 \gamma}}\right) .
$$


Ignoring the result obtained in gas, the discrepancy originally obtained in the Ann Arbor experiment could be explained if $\epsilon \approx 10^{-7}$.

\subsection{Mirror world and dark matter}

Foot [6 discussed the possible importance of mirror matter in the context of the dark matter puzzle: if mirror matter is identified with the dark matter in the Universe, then it is natural to interpret the dark matter halo of our galaxy as containing mirror matter (possibly mirror stars/planets/dust and gas [6]). If the dark matter halo of our galaxy is composed of mirror matter, then it can potentially be detected in dark matter experiments via the nuclear recoil signature via interactions between nuclei and mirror-nuclei induced by the photon-mirror photon kinetic term mixing.

Very strikingly Foot recently pointed out 14 that the DAMA results 15 could be interpreted in terms of such interactions, if one interprets the signal in terms of mirror $O^{\prime}, F e^{\prime}$ mixture with an annual modulation effect in the $2-6 \mathrm{keV}$ window:

$$
|\epsilon| \sqrt{\frac{\xi_{O^{\prime}}}{0.10}+\frac{\xi_{F e^{\prime}}}{0.02}} \simeq 4.5 \times 10^{-9}
$$

where $\xi_{A^{\prime}} \equiv \rho_{A^{\prime}} /\left(0.3 \mathrm{GeV} / \mathrm{cm}^{3}\right)$ is the $A^{\prime}$ proportion (by mass) of the halo dark matter. This is an extremely impressive result which provides an indication for possible values of $\epsilon$ ! These are summarized in Figure 2 by Foot $[\underline{6}$. An interesting region, which seems also to be in the range of naturally small $\epsilon$ motivated by Grand Unification models as discussed by Berezhiani[5], is $10^{-9} \lesssim \epsilon \lesssim 10^{-8}$. Using Eq 5 this implies $10^{-8}<\operatorname{Br}(o-P s \rightarrow$ invisible $)<10^{-6}$.

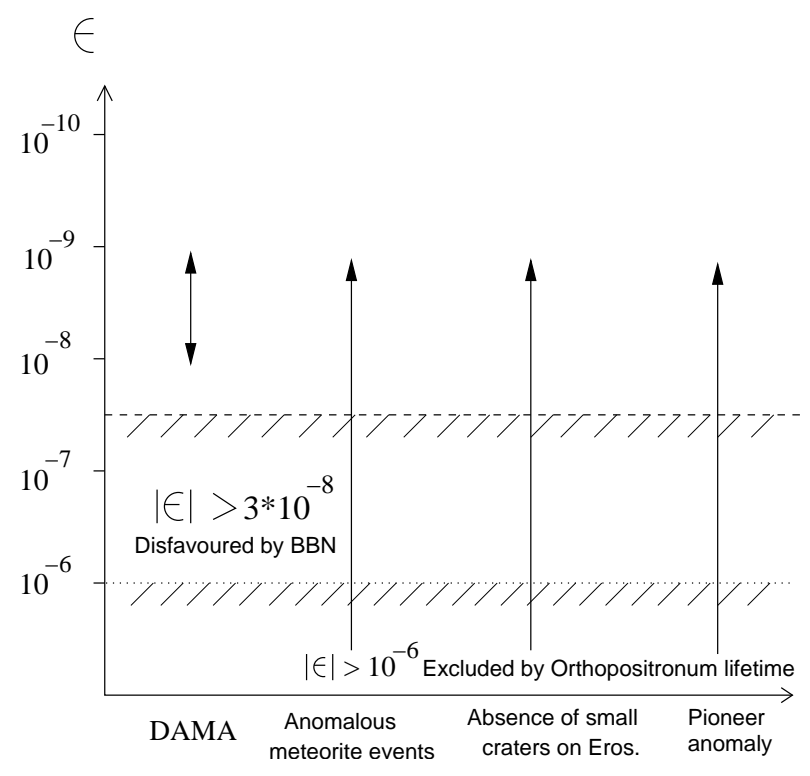

Figure 2: Summary of the experimental constraints for $\epsilon$ by Foot [[6].

\subsection{Cosmology of mirror matter}

How does the picture of mirror matter as dark matter candidate fit in the global picture of the evolution of the Universe as predicted by cosmology? Berezhiani $[5$ brought up important points concerning these issues: naively mirror parity implies that ordinary and mirror sectors should have the same cosmology, 
and so ordinary and mirror-particles should have the same cosmological densities. However, this would be in the immediate conflict with the Big Bang nucleosynthesis (BBN) bounds on the effective number of extra light neutrinos. Therefore, the mirror particle density in the early universe should be appropriately reduced. This situation is plausible if the following conditions are satisfied [5]:

A. At the Big Bang the two systems are born with different densities.

B. The two systems interact very weakly, so that they do not come into the thermal equilibrium with each other in the early Universe.

C. Both systems expand adiabatically and there is no significant entropy production at the later times which could heat the mirror-sector and equilibrate its temperatures to the ordinary one.

Because of the temperature difference, in the mirror sector all key epochs as the baryogenesis, nucleosynthesis, etc. proceed at somewhat different conditions than in the observable universe. In particular, in certain baryogenesis scenarios the mirror-world generically should get a larger baryon asymmetry than the ordinary sector, and it is pretty plausible that dark matter of the Universe or at least its significant fraction, is constituted by mirror-baryons which are obviously dark for the ordinary observer [16.

\section{Extra space-time dimensions and invisible decays of positro- nium}

Recently the models with infinite additional dimensions of the Randall-Sundrum type (brane-world models) have become very popular. There is a hope that models with a big compactification radius will provide the natural solution to the gauge hierarchy problem. For instance, as it has been shown by Randall and Sundrum in the five dimensional model, there exists a thin-brane solution to the 5-dimensional Einstein equations which has flat 4-dimensional hypersurfaces,

$$
d s^{2}=a^{2}(z) \eta_{\mu \nu} d x^{\mu} d x^{\nu}-d z^{2}
$$

Here

$$
a(z)=\exp \left(-k\left(z-z_{c}\right)\right)
$$

and the parameter $k>0$ is determined by the 5-dimensional Planck mass and bulk cosmological constant. For the model with metric of Randall-Sundrum, the effective four-dimensional gravitational constant is

$$
G_{(4)}=G_{(5)} k \frac{1}{\exp \left(2 k z_{c}\right)-1}
$$

One can solve the gauge hierarchy problem in this model if $k \sim M_{E W}=1 T e V, G_{(5)} \sim k^{-3}$. It follows that the Planck scale in this model is

$$
M_{P L} \sim \exp \left(k z_{c}\right) M_{E W}
$$

that means the existence of exponential hierarchy between Planck and electroweak scales. For $z_{c} \approx 37 \cdot k^{-1}$ we have a correct quantitative relation between Planck and electroweak scales.

In Randall-Sundrum models, physical particles are trapped on a three-dimensional brane via curvature in the bulk dimension. Although massive particles can indeed be trapped on the brane, they are also expected to be metastable[17]. That is, the quasi-normal modes are metastable states, that can decay into continuum Kaluza-Klein modes in the higher dimensions. For an observer on the three-brane, massive particles will appear to exist for some time and then "disappear" into the bulk fifth dimension.

It was noted 18] that $o-P s$ is a good candidate for searching for this effect of disappearance into additional dimension(s) since it has specific quantum numbers similar to those of the vacuum and is a 
system which allows its constituents a rather long interaction time. For the orthopositronium invisible decay into additional dimension(s)

$$
o-P s \rightarrow \gamma^{*} \rightarrow \text { additional dimension }(s)
$$

the corresponding branching ratio is 18

$$
\begin{gathered}
\operatorname{Br}\left(o-P s \rightarrow \gamma^{*} \rightarrow \text { additional dimension }(s)\right)= \\
\frac{9 \pi}{4\left(\pi^{2}-9\right)} \cdot \frac{1}{\alpha^{2}} \cdot \frac{\pi}{16}\left(\frac{m_{o-P s}}{k}\right)^{2} \approx 3 \cdot 10^{4}\left(\frac{m_{o-P s}}{k}\right)^{2}
\end{gathered}
$$

An important bound on the parameter $k$ arises from data on the $Z \rightarrow$ invisible decay, which leads to $k \gtrsim 2.7 \mathrm{TeV}$. Using this, we find

$$
\operatorname{Br}(o-P s \rightarrow \text { additional dimension }(s)) \lesssim 4 \cdot 10^{-9}
$$

To solve the gauge hierarchy problem models with additional infinite dimension(s) must have the $k \lesssim$ $O(10) \mathrm{TeV}$. It means that

$$
\operatorname{Br}(o-P s \rightarrow \text { additional dimension }(s)) \gtrsim O\left(10^{-10}\right)
$$

Since these estimates give only an order of magnitude for the lower and upper limits on corresponding branching ratios, we believe that the region of $\operatorname{Br}(o-P s \rightarrow$ invisible $) \simeq 10^{-9}-10^{-8}$ is of great interest for an observation of the effect of extra dimensions.

\section{Orthopositronium decay rate puzzle}

Measurements of the positronium decays have a long history. The measurements of the o-Ps lifetime performed after 1987 are summarized in Table 2] Three precision measurements 19, 11, 12 of the o-Ps decay rate were originally performed at Ann Arbor, which reported decay rate values much larger, i.e., 5.1 - 8.9 experimental standard deviations, than the QED prediction 23. This discrepancy has been referred to as 'orthopositronium lifetime puzzle', and was a long-standing problem. Measurements performed in Tokyo 13, 20, 22 and the recent Ann Arbor measurement 21] are in agreement with theory. Experiments differed substantially in the technique of formation of o-Ps.

Table 2: Experimental results and theory (from Sillou[24]).

\begin{tabular}{lcccccc}
\hline Year & group/ref & $\begin{array}{c}\text { Rate } \\
\mu s^{-1}\end{array}$ & $\begin{array}{c}\text { errors } \\
(\mathrm{ppm})\end{array}$ & Technique & exp - th & $\begin{array}{c}\text { exp - th } \\
(\text { sigma })\end{array}$ \\
\hline 1987 & Ann Arbor [19] $]$ & 7.0516 & 180 & Gas & .01162 & 8.9 \\
1989 & Ann Arbor [11] & 7.0514 & 200 & Gas & .01142 & 8.2 \\
1990 & Ann Arbor[12]] & 7.0482 & 230 & Vacuum & .00822 & 5.1 \\
1995 & Tokyo [[13]] & 7.0398 & 412 & Powder & -.00018 & -.06 \\
2000 & Tokyo [[20]] & 7.0399 & 412 & Powder & -.00008 & 0.0 \\
2003 & Ann Arbor [[21] $]$ & 7.0404 & 185 & Vacuum & .00042 & 0.32 \\
2003 & Tokyo [[22]] & 7.0396 & 227 & Powder & -.00038 & .024 \\
2000 & AFS[[23] & 7.039979 & 1.6 & Theory & & \\
\hline
\end{tabular}

To elucidate discrepancies, a variety of experiments have been carried out to search for the exotic decay mode of o-Ps, resulting in no evidence [25, 26, 27, 28, 29, 30, 31]. Some of these searches are reported in Table 3 Note that best present limit for the branching ratio for invisible channels of $o-P s$ is 27 .

$$
\operatorname{Br}(o-P s \rightarrow \text { invisible })<2.8 \times 10^{-6}
$$


Table 3: Upper limits on the branching ratios of several exotic o-Ps decays.

\begin{tabular}{cccc}
\hline \hline Decay Mode & $90 \%$ upper limit & Comments & Group \\
\hline$\gamma+X$ & $1.1 \mathrm{ppm}$ & $\begin{array}{c}X \text { long-lived boson } \\
\mathrm{m}_{X}<800 \mathrm{keV}\end{array}$ & $\begin{array}{c}\text { CERN, Moscow, } \\
\text { Tokyo, Heidelberg }\end{array}$ \\
\hline$\gamma+X \rightarrow \gamma+2 \gamma$ & $400 \mathrm{ppm}$ & $\begin{array}{c}\text { Short-lived boson } \\
\mathrm{m}_{X}<900 \mathrm{keV}\end{array}$ & $\begin{array}{c}\text { Moscow, } \\
\text { Tokyo }\end{array}$ \\
\hline$\gamma \gamma$ & $3.5 \mathrm{ppm}$ & $\begin{array}{c}\text { Forbidden by angular } \\
\text { momentum conservation }\end{array}$ & Ann Arbor, \\
& $2.6 \mathrm{ppm}$ & Forbidden by C-parity & \\
\hline$\gamma \gamma \gamma \gamma$ & $44 \mathrm{ppm}$ & $\mathrm{m}_{X_{1}}+\mathrm{m}_{X_{2}}<900 \mathrm{keV}$ & ETHZ- Moscow \\
\hline$\gamma+X_{1}+X_{2}$ & $2.8 \mathrm{ppm}$ & Not in vacuum & Moscow, Tokyo \\
\hline Invisible & & &
\end{tabular}

However, this result was obtained in powder, and hence some of the comments expressed in Section 2.1 might be relevant 32 . As long as $t \gg 1 / \Gamma_{\text {coll }}$, we have

$$
\Gamma^{o b s} \simeq \Gamma_{3 \gamma}\left(1+\frac{2 \omega^{2}}{\Gamma_{c o l l} \Gamma_{3 \gamma}}\right) .
$$

The difference between the higher decay rate measured in the vacuum cavity experiment, relative to the value predicted by theory, can be expressed as

$$
\Gamma_{e x p}-\Gamma_{3 \gamma} \simeq \frac{2 \omega^{2}}{\Gamma_{c o l l} \Gamma_{3 \gamma}}
$$

For $\Gamma_{\text {coll }} \lesssim 3 \Gamma_{3 \gamma}$, which should be applicable to the Ann Arbor cavity experiment (see Table 1), one finds that

$$
\omega^{2} \sim 2 \times 10^{-3} \Gamma_{3 \gamma}^{2} \Rightarrow \epsilon \lesssim 10^{-6} .
$$

Thus, the limit of Eq.(21) is still not strong enough compared to the BBN (see Figure 2).

Note, that the Tokyo results 13, 20, 22, and measurements in vacuum 12, 21] are still consistent with the hypothesis of the mirror matter. Indeed in the Tokyo experiment the effect is suppressed by the very large collision rate of o-Ps in the powder, so it is irrelevant. The contribution of the mirror matter effect to the o-Ps decay rate in vacuum is allowed to be at the level of less than $\Delta \Gamma_{\text {mirror }} \simeq 100 p p m$ according to the BBN limit on $\epsilon$. This is consistent with an "exotic" contribution to the o-Ps decay rate, which is allowed to be $\Delta \Gamma_{\text {exotic }}<\Gamma_{\text {Michigan }}-\Gamma_{Q E D} \simeq 400 p p m$, as one can derive from the difference between the new Michigan result and AFS calculations, see Table 2

\subsection{Status of the QED calculations}

Theoretical aspects in theoretical predictions of quantities related to positronium have been summarized at this workshop by Karschenboim 33, Penin 34] and Smith 35.

Positronium, an electromagnetic bound state of the electron $e^{-}$and the positron $e^{+}$, is the lightest known atom. Thanks to the smallness of the electron mass $m_{e}$ the strong and weak interaction effects are negligible and its properties can be calculated perturbatively in quantum electrodynamics (QED) as an expansion in Sommerfeld's fine structure constant $\alpha$ with very high precision only limited by the complexity of the calculations. Positronium is thus a unique laboratory for testing the QED theory of bound systems. The theoretical analysis is, however, complicated in comparison to other hydrogen-like atoms by annihilation and recoil effects. At the same time due to negligible short-distance effects of the virtual strongly interacting heavy particles, positronium could be a sensitive probe of the "new physics" at long distance. 
The present theoretical knowledge of the decay rates (widths) of the ${ }^{3} S_{1}$ orthopositronium (o-Ps) and ${ }^{1} S_{0}$ parapositronium (p-Ps) ground states to two and three photons, respectively, may be summarized as follows 34 :

$$
\begin{aligned}
\Gamma_{o}^{\mathrm{th}}= & \frac{2\left(\pi^{2}-9\right) \alpha^{6} m_{e}}{9 \pi}\left\{1+\frac{\alpha}{\pi} 10.286606(10)+\left(\frac{\alpha}{\pi}\right)^{2}\left[\frac{\pi^{2}}{3} \ln \alpha+44.87(26)\right]\right. \\
& \left.+\frac{\alpha^{3}}{\pi}\left[-\frac{3}{2} \ln ^{2} \alpha+\left(3.428869(3)-\frac{229}{30}-8 \ln 2\right) \ln \alpha+\frac{D_{o}}{\pi^{2}}\right]\right\}, \\
\Gamma_{p}^{\mathrm{th}}= & \frac{\alpha^{5} m_{e}}{2}\left\{1+\frac{\alpha}{\pi}\left(\frac{\pi^{2}}{4}-5\right)+\left(\frac{\alpha}{\pi}\right)^{2}\left[-2 \pi^{2} \ln \alpha+5.1243(33)\right]\right. \\
& \left.+\frac{\alpha^{3}}{\pi}\left[-\frac{3}{2} \ln ^{2} \alpha+\left(\frac{533}{90}-\frac{\pi^{2}}{2}+10 \ln 2\right) \ln \alpha+\frac{D_{p}}{\pi^{2}}\right]\right\} .
\end{aligned}
$$

The coefficients $D_{o, p}$ parameterize the unknown nonlogarithmic $\mathcal{O}\left(\alpha^{3}\right)$ terms. The calculation of missing $\mathcal{O}\left(\alpha^{3}\right)$ nonlogarithmic terms would be one of the most complicated perturbative calculations in quantum field theory though conceptually the problem is clear, all the necessary tools are at hand and a number of partial results have been obtained. Currently the experimental uncertainty exceeds the theoretical one by two orders of magnitude for positronium decay rates and by a factor of two for HFS. A lifetime experiment with an accuracy better than $50 \mathrm{ppm}$ would be able to test the second-order correction which is $\simeq 45\left(\frac{\alpha}{\pi}\right)^{2} \approx 200$ ppm. New measurements of much higher accuracy would be mandatory to unambiguously confirm or confront the QED predictions to higher-levels and to inspire the theorists to complete the $\mathcal{O}\left(\alpha^{3}\right)$ computations.

\subsection{Orthopositronium decay rate measurements}

As some fraction of o-Ps inevitably results in 'pick-off' annihilations due to collisions with atomic electrons of the target material, the observed o-Ps decay rate $\lambda_{o b s}$ is a sum of the intrinsic o-Ps decay rate $\lambda_{\text {o-Ps }}$ and the pick-off annihilation rate into $2 \gamma$ 's, $\lambda_{\text {pick }}$, i.e.,

$$
\lambda_{\text {obs }}(t)=\lambda_{3 \gamma}+\lambda_{\text {pick }}(t) .
$$

$\lambda_{\text {pick }}(t)$ is proportional to the rate of o-Ps collisions with the target materials, i.e.; $\lambda_{\text {pick }}=n \sigma_{a} v(t)$, where $n$ is product of the density of the target, $\sigma_{a}$ the annihilation cross-section, and $v(t)$ the time dependent velocity of o-Ps. Due to the thermalization process of o-Ps, this necessitates expressing $\lambda_{\text {pick }}$ as a function of time whose properties are dependent on the surrounding materials. Thermalization process should be carefully treated even in the cavity experiment 12. Although pickoff correction is small in cavities, disappearance of o-Ps through the cavity entrance aperture has large contribution to $\lambda_{o b s}$. This disappearance rate is also proportional to $v(t)$, as the same reason. Since the rate of elastic collision is extremely small in cavities, it takes much time, longer than $1 \mu \mathrm{s}$, to thermalize well, and the disappearance rate still depends strongly on time.

In the Ann Arbor measurements 19, 11, 12, $\lambda_{\text {obs }}$ 's were measured by varying the densities of the target materials, size of the cavities and also the entrance aperture of the cavities. The extrapolation to zero density or aperture was expected to yield the decay rate in a vacuum, $\lambda_{3 \gamma}$, under the assumption of quick thermalization (shorter than 170-180 nsec) with constant o-Ps velocity. However, this assumption contains a serious systematic error as pointed out in reference 36, 37, 38.

The Tokyo group proposed an entirely new method 37, which is free from above-mentioned systematic error. The energy distribution of photons from the 3-body decay is continuous below the steep edge at $511 \mathrm{keV}$, whereas the pick-off annihilation is 2-body which produces a $511 \mathrm{keV}$ monochromatic peak. Energy and timing information are simultaneously measured with high-energy resolution germanium detectors such that $\lambda_{\text {pick }}(t) / \lambda_{3 \gamma}$ can be determined from the energy spectrum of the emitted photon. 
Once a precise thermalization function is obtained, $\lambda_{\text {pick }}(t)$ will contain all information about the process. The population of o-Ps at time $t, N(t)$ can be expressed as

$$
N(t)=N_{0}^{\prime} \exp \left(-\lambda_{3 \gamma} \int_{0}^{t}\left(1+\frac{\lambda_{p i c k}\left(t^{\prime}\right)}{\lambda_{3 \gamma}}\right) d t^{\prime}\right) .
$$

Providing the ratio is determined as a function of time, the intrinsic decay rate of o-Ps, $\lambda_{3 \gamma}$, can be directly obtained by fitting the observed time spectrum (See Figure 3 ).

Asai et al. obtained the decay rate of 7.0398(29) and 7.0399(25) $\mathrm{ss}^{-1}$ independently 37, 20, which are consistent with the non-relativistic QED calculation[23], and quite differ from the results originally obtained at Ann Arbor 19, 11, 12, 7.0482(16)-7.0516(13) $\mu \mathrm{s}^{-1}$. The 2003 Ann Arbor result is 7.0404(13) $\mu \mathrm{s}^{-1}$.

The observed $\lambda_{\text {pick }}(t)$ indicates that o-Ps thermalization is slow and it is a serious systematic problem in all experiments using an extrapolation. In 1998, the Ann Arbor group recognized that the incomplete thermalization can have serious consequences in their measurements 39, but they did not yet update the orthopositronium lifetime.

Regardless, still several issues existed in the first results of Asai et al. 38]: (i) accuracy was $350 \mathrm{ppm}$, being worse than those of the other experiments 19, 11, 12, (ii) there were unknown systematic uncertainties before $t_{\text {start }}=200 \mathrm{~ns}$, and therefore to remove this uncertainty, final results were obtained using data after $220 \mathrm{~ns}$, and (iii) systematic error regarding the Stark effect was not estimated. Improving results by considering these problems has been contributed to these proceedings, where the final result using this new method were reported 38 . The obtained decay rates are $\lambda_{3 \gamma}=7.03991 \pm 0.0017$ (stat.) $\mu s^{-1}$ for RUN 1 and $7.03935 \pm 0.0017$ (stat.) $\mu s^{-1}$ for RUN 2, which are consistent with each other. It turns out that the thermalization process and pickoff ratio are different within each run, however, excellent agreement between two runs is obtained. This should indicate that the method correctly takes into account thermalization and pickoff correction.

\subsection{A personal summary}

Since the improvement of the o-Ps decay rate in vacuum by the new calculations 23, the theoretical error is two orders of magnitude smaller than the experimental one. One could therefore only agree with the conclusion from the latest AFS paper [23: "obviously, no conclusion can be drawn until the experimental situation is clarified".

The current experimental situation concerning the measurements of the orthopositronium decay rate is graphically summarized in Figure 4 where the decay rate is plotted as a function of the year of the measurement. The last point is the theoretical value. Different experiments have different systematic problems.

The much excitement that arose when the measurements performed by the Michigan group first in gas then in vacuum did not agree with theory seemed to have vanished since the Michigan group has published a new result which is now in agreement with the theoretical value, somewhat in contradiction with their earlier results. The easy shortcut is to assume that the o-Ps-lifetime puzzle is closed. Should we however indeed consider that the o-Ps lifetime puzzle is solved and hence assume that further experimental investigations with positronium are irrelevant?

In the following, we play the Devil's advocate and list a few comments relative to the Ann Arbor experiments:

- The Michigan group concludes that they did not take properly into account the "backscattered" high energy positronium component. 

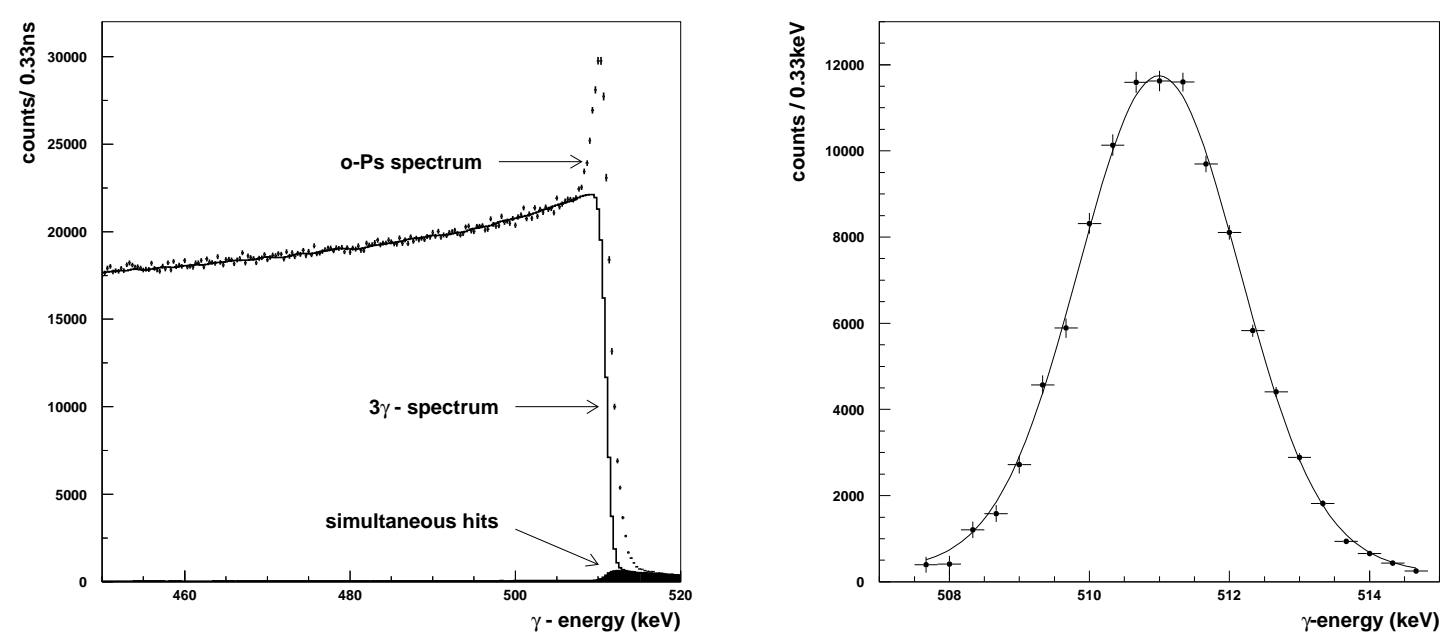

Figure 3: Tokyo experiment (Figures from Asai[38]): (a) Energy spectrum of o-Ps decay $\gamma$ 's obtained by Ge detectors. Dots represent data points in a time window of $150-700 \mathrm{~ns}$, and the solid line shows the $3 \gamma$-decay spectrum calculated by Monte Carlo simulation. Shaded area indicate simultaneous hits estimated by the simulation. (b) Pick-off spectrum obtained after subtracting the $3 \gamma$ contribution from the o-Ps spectrum. The solid line represents the fit result.

- This interpretation seems to be in contradiction with the results of an experiment performed with the same setup to search for a $2 \gamma$ decay channel of o-Ps 40. The energy spectrum near $511 \mathrm{keV}$ measured with a precision Ge detector is shown in Figure [5 No evidence for $2 \gamma$ events (pickoff signal) is visible. They exclude these decays at the level of $\simeq 200 \mathrm{ppm}$, which is a factor 5 smaller than the original discrepancy.

Similarly, a certain number of comments can be addressed to the Tokyo experiments:

- Systematic errors give a substantial contribution to the measurement error. In particular, the correction due to pickoff in the high density target is large 38]:

$$
\lambda_{\text {pick }}(t>200 n s) \approx 0.01 \times \lambda_{3 \gamma}=\left(10^{4} \text { ppm }\right) \times \lambda_{3 \gamma}
$$

while the original discrepancy was at the level of $1000 \mathrm{ppm}$. Hence, the correction is an order of magnitude larger than the original effect.

- The experimental data seemed to be too consistent with theory. Indeed, the difference between experiment and theory in sigmas are resp. $-0.06,0.0$ and 0.024 for the resp. 1995, 2000 and 2003 measurements. The statistical probability to obtain three measurements within $0.06 \sigma$ is $\approx 10^{-4}$. The origin of this could be related to the fact that measurements are systematic dominated. Clearly, many of these errors are correlated and as a result the various measurements cannot be easily combined.

The naive outcome from this non-exhaustive list is that new experiments to measure the orthopositronium decay rate in vacuum in which the pickoff rate can be measured directly via $2 \gamma$ decays would bring new valuable information. 
In addition, the current level of the theoretical precision is about two orders of magnitude better than the experimental one. Thus, further positron beam based experiments to measure the $o-P s$ decay rate in vacuum are required and are of great interest to test high-order QED corrections. In general, the tendency is to go towards high granularity hermetic detector in order to also discriminate on event topologies. This allows simultaneous fit of time distributions in conjunction with simple topological cuts.

In parallel, other experimental approaches like the direct search for invisible decays of o-Ps should be pursued, as discussed in Section 6

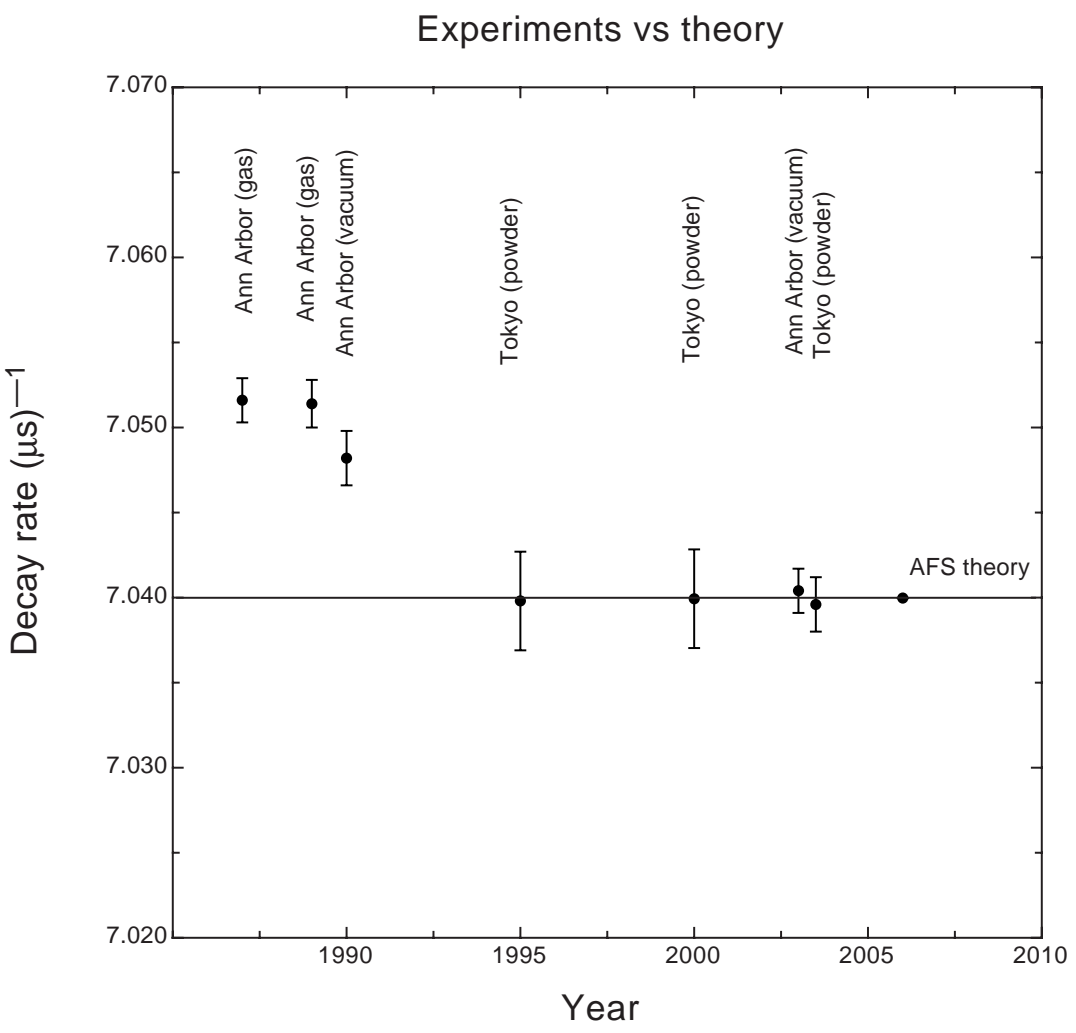

Figure 4: Summary of the experimental results on o-Ps-lifetime measurements as a function of the year. The last point is the theoretical value from AFS[23].

\section{$5 \quad$ Positronium spectroscopy - hyperfine splitting}

Positronium HFS, $\Delta \nu=E\left(1^{3} S_{1}\right)-E\left(1^{1} S_{0}\right)$, where $E\left(1^{1} S_{0}\right)$ and $E\left(1^{3} S_{1}\right)$ are the energy levels of p-Ps and o-Ps ground state, is the most precisely measured quantity in positronium spectroscopy as far as the absolute precision is concerned however measurements are not recent 41, 42. On the theoretical side we have

$$
\begin{aligned}
\Delta \nu^{\text {th }}= & \frac{7 m_{e} \alpha^{4}}{12}\left\{1-\frac{\alpha}{\pi}\left(\frac{32}{21}+\frac{6}{7} \ln 2\right)+\left(\frac{\alpha}{\pi}\right)^{2}\left[-\frac{5}{14} \pi^{2} \ln \alpha+\frac{1367}{378}-\frac{5197}{2016} \pi^{2}\right.\right. \\
& \left.+\left(\frac{6}{7}+\frac{221}{84} \pi^{2}\right) \ln 2-\frac{159}{56} \zeta(3)\right]+\frac{\alpha^{3}}{\pi}\left[-\frac{3}{2} \ln ^{2} \alpha-\left(\frac{62}{15}-\frac{68}{7} \ln 2\right) \ln \alpha\right. \\
& \left.\left.+\frac{D_{\nu}}{\pi^{2}}\right]\right\} .
\end{aligned}
$$




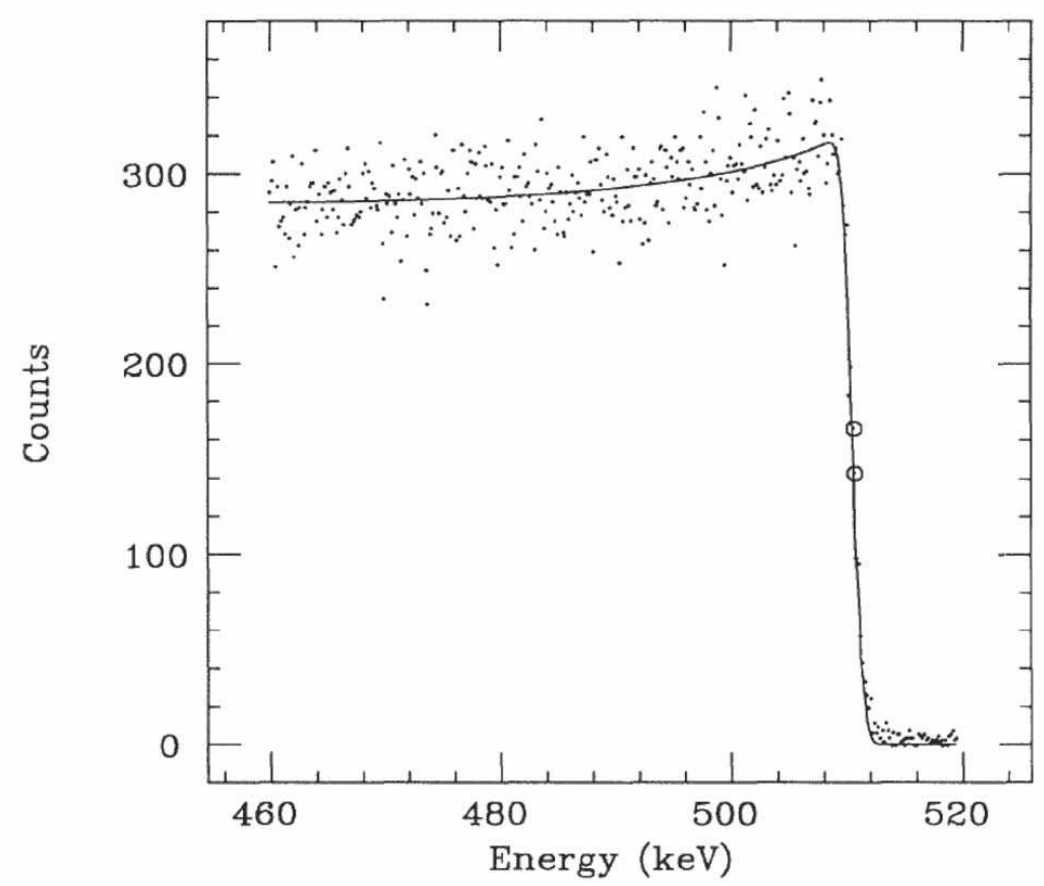

Figure 5: Ann Arbor experiment (from Gidley et al.[40]): Energy spectrum near $511 \mathrm{KeV}$ measured with a precision Ge detector. No evidence for $2 \gamma$ events (pickoff signal) is visible.

The theoretical result exceeds by approximately 2.6 and 3.5 experimental standard deviations (see Figure 6). On the other hand, the measured $1 S-2 S$ interval seems to be in agreement with theory. This shows that the understanding of the positronium spectroscopy is not satisfactory.

Hence, new experiments on spectroscopy of positronium would bring new valuable information. These experiments are however very different than those to measure the lifetime or search for invisible decay. There are midway between particle and atomic physics experiment and require in addition expertise in the field of lasers and optics. We are not aware of any plans for such an experiment.

\section{New searches for invisible decay of positronium}

As already mentioned in the previous sections, the new models that are relevant to the $o-P s \rightarrow$ invisible decay mode predict (see Ref. 43] for a review) the existence either of i) extra-dimensions, or ii) fractionally charged particles, or iii) a new light vector gauge boson, or iv) dark matter of the mirror matter type. The required sensitivity in the branching ratio $\operatorname{Br}(o-P s \rightarrow$ invisible $)$ for the possible observation of these phenomena has to be at least as low as $10^{-8}$.

\subsection{ETHZ-INR experiment in silica aerogel}

A new experiment, aimed at developing a $4 \pi$ hermetic calorimeter with an extremely low photon detection inefficiency, was presented by Crivelli at this workshop [45]. The main components of the detector are: the positron source $\left({ }^{22} \mathrm{Na}\right)$, the positron tagging system, composed of a scintillating fiber viewed by two photomultipliers (PM), the positronium formation $\mathrm{SiO}_{2}$ target and a hermetic $\gamma$-detector. The coincidence of the PM signals from the positrons crossing the fiber, opens the gate for the data acquisition 


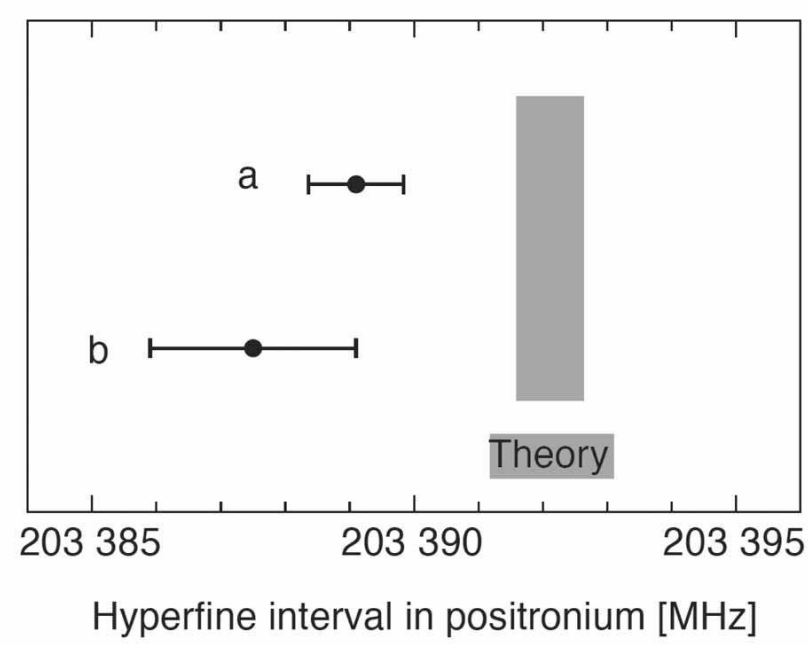

Figure 6: The positronium $1 S$ hyperfine splitting: a comparison of theory to experiments. Figure from Ref. 33].

(DAQ). In the off-line analysis the $1.27 \mathrm{MeV}$ photon, which is emitted from the source simultaneously with the positron, is required to be in the trigger BGO counter resulting in a high confidence level of positron appearance in the positronium formation region. A positron, which enters the $\mathrm{SiO}_{2}$ target may capture an electron creating positronium. The calorimeter detects, either the direct $2 \gamma$ annihilation in flight or the 2(3) photons from the para (ortho)-positronium decays in the target. The occurence of the $o-P s \rightarrow$ invisible decay would appear as an excess of events with deposition in the calorimeter compatible with zero above those expected from the Monte Carlo prediction or from the direct background measurement. This measurement presents a new feature of this type of experiment. The idea is to obtain a pure o-Ps decay energy spectrum by comparing two different spectra from the same target filled either with $\mathrm{N}_{2}$ (low o- Ps quenching rate) or with air, where the presence of paramagnetic $\mathrm{O}_{2}$ will quench the fraction of o-Ps in the target from $10 \%$ down to $3 \%$, due to the spin exchange mechanism:

$$
o-P s+O(\uparrow \uparrow) \rightarrow p-P s+O(\downarrow \uparrow)
$$

Thus the subtraction of these properly normalized spectra will result in a pure o-Ps annihilation energy spectrum in the $\gamma$-detector.

The experiment is currently in commissioning phase (see Figure [7). Results are expected in 2004.

\subsection{ETHZ-INR experiment in vacuum}

The concepts for a new experiment, designed with the goal to observe the $o-P s \rightarrow$ invisible decays, if its branching ratio is greater than $10^{-7}$, were presented by Gninenko at this workshop [46]. Figure 8 shows a schematic view of the experimental setup.

Accordingly, the apparatus is designed with several distinct parts: i) a pulsed slow positron beam and a low-mass target for efficient orthopositronium production in a vacuum cavity, ii) a positron appearance tagging system with a high signal-to-noise ratio based on a high performance MCP, iii) an almost $4 \pi$ BGO crystal calorimeter (ECAL) surrounding the vacuum cavity for efficient detection of annihilation photons. The cavity has as little wall mass as possible to minimize photon energy absorption.

The occurrence of the $o-P s \rightarrow o-P s^{\prime} \rightarrow$ invisible conversion would appear as an excess of events with energy deposition comparable with zero in the calorimeter above those expected from the prediction 


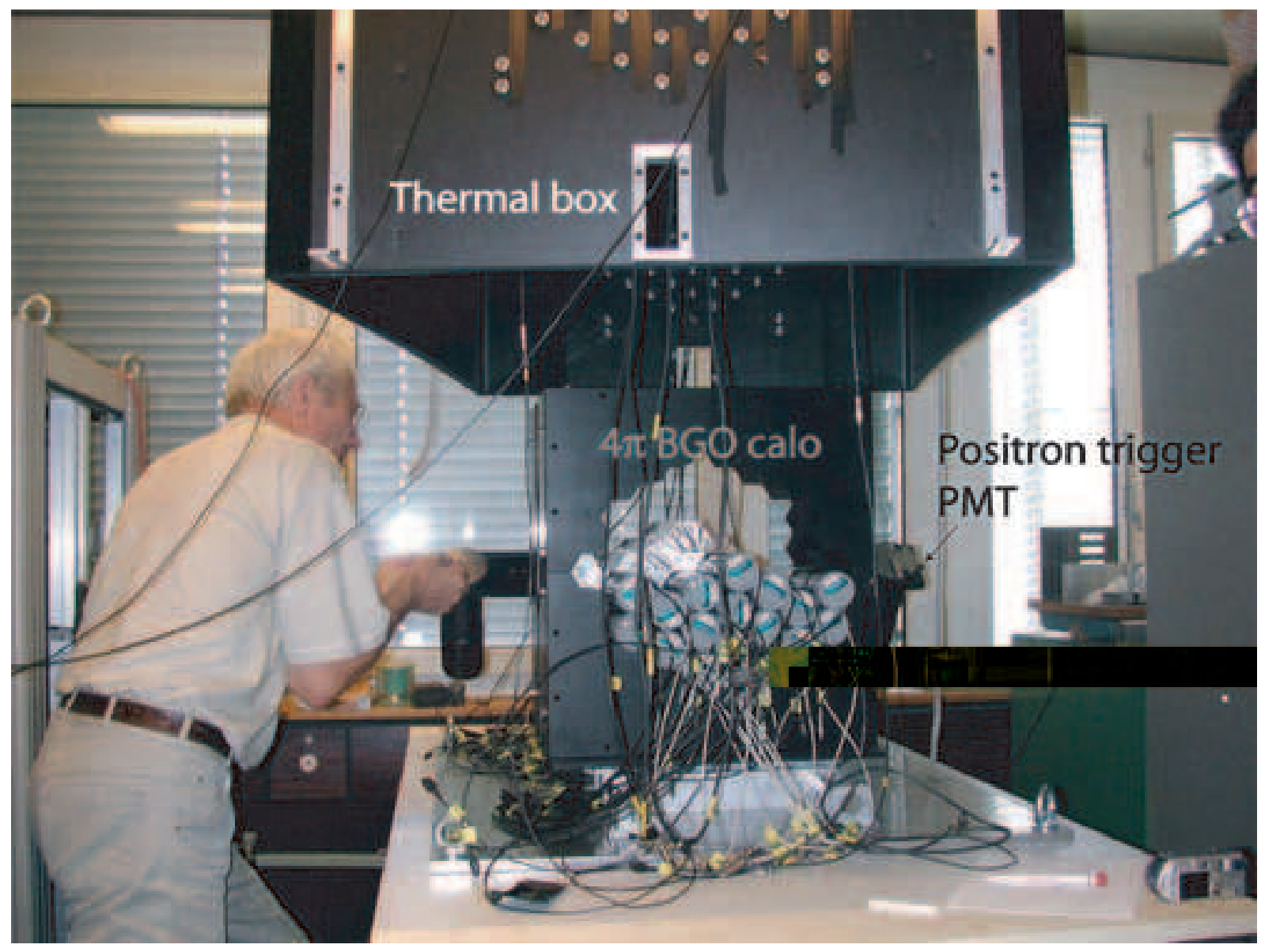

Figure 7: ETHZ-INR experiment for o-Ps $\rightarrow$ invisible decay search in silica aerogel.

of the background. In case of a signal observation the number of excess events could be cross-checked by small variations of experimental conditions which affect the $o-P s \rightarrow o-P s^{\prime}$ transition rate but do not result in a loss of energy from ordinary positron annihilations. The identification of signal events relies on a high-efficiency measurement of the energy deposition from the annihilation of positrons.

To achieve a sensitivity in the branching ratio of $10^{-7}$ in a reasonable amount of data-taking time, the rate of $o-P s$ decays per second has to be as high as possible consistent with minimal reduction of the $o-P s \rightarrow$ invisible signal efficiency and acceptably small dead time. For the pulsed positron beam design presented by Gninenko at this workshop [4], the trigger rate in the photon detector is expected to be $\simeq 100 \mathrm{~Hz}$ which is low enough to allow these events to be recorded without losses, and is high enough to reach the expected sensitivity (see below) in a reasonable time.

Positrons from the pulsed beam are stopped in the $\mathrm{MgO}$ target and either form positronium, i.e. $o-P s$ or $p-P s$, or annihilate promptly into $2 \gamma$ 's. The secondary electrons (SE) produced by the positrons hitting the target are accelerated by the voltage applied to the target relative the grounded transport tube. Then they are transported by a magnetic field in the backward direction relative to the positrons moving in spirals along the magnetic field lines and deflected to a microchannel plate (MCP) by a $E \times B$ filter.

The trigger for data acquisition is generated by a coincidence within $\pm 3 \mathrm{~ns}$ of a pulse from the MCP and the signal from the pulsed beam, which is synchronized with the positron arrival time at the target. 

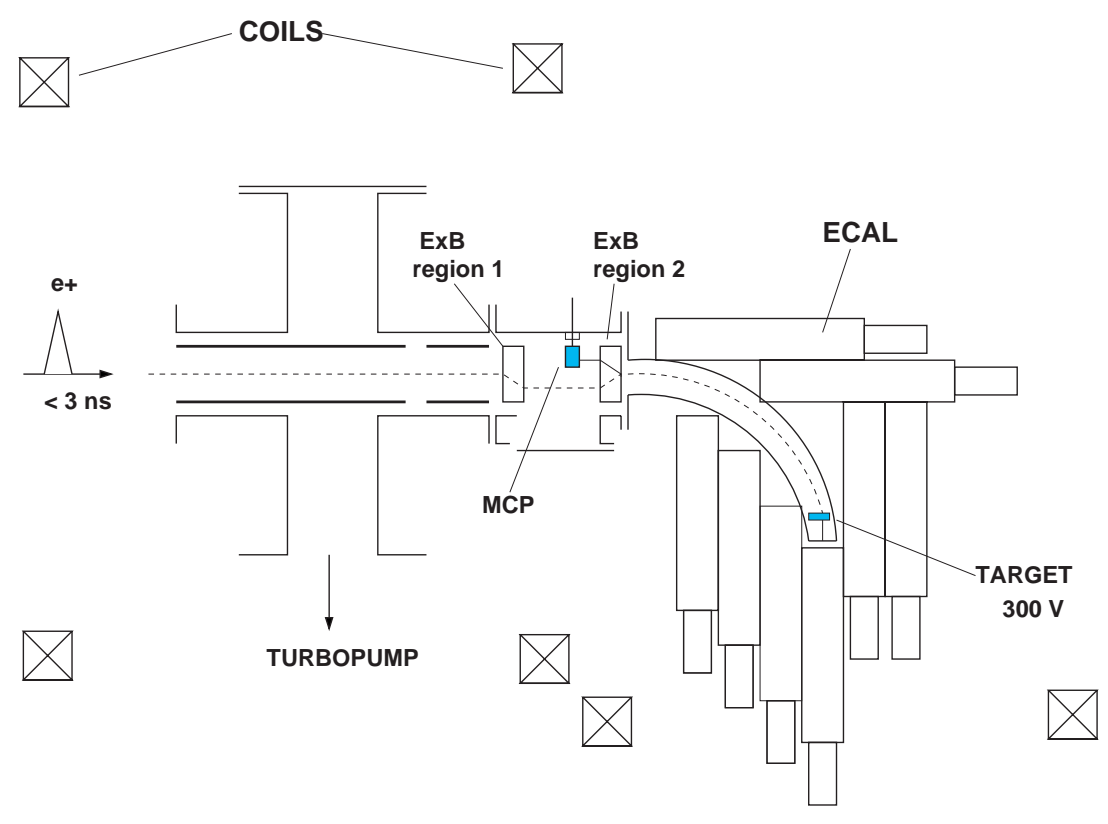

Figure 8: Schematic diagram of the ETHZ-INR experimental setup.

It is hoped that the beam can serve several different experiments. Thus, the final beam construction should compromise several design goals which are summarized as follows:

- beam energy range from $100 \mathrm{eV}$ to $1000 \mathrm{eV}$,

- beam intensity of $\simeq 10^{4}-10^{5}$ positrons per second,

- pulse duration at the target $\delta t_{T}<3 \mathrm{~ns}$ for an initial pulse duration at the moderator $\delta t_{M} \simeq 300-400$ ns,

- repetition rate $0.3-1.0 \mathrm{MHz}$,

- high peak/noise ratio, (single) Gaussian shape of the pulse,

- beam spot size at the target position is of the order of a few millimeters assuming $3-5 \mathrm{~mm}{ }^{22} \mathrm{Na}$ source diameter,

Figure 9 shows schematic illustration of the pulsed positron beam design. The positron pulsing section consists of a chopper and a buncher and is based on positron velocity modulation combined with the RF bunching technique. A positive potential $(\simeq 100 \mathrm{eV})$ is applied to the moderator foil in order to insure the proper energy of the positrons at the buncher input. Initial positron pulses with duration 300 ns are formed with the chopper grid placed $2 \mathrm{~mm}$ apart from the moderator foil. The pulsed voltage with an amplitude of about $+5 \mathrm{~V}$ applied to the chopper grid relative the moderator foil will stop slow positrons with energy about $3 \mathrm{eV}$ emitted from the moderator. Fast positrons emitted from the source are eliminated from the beam by the velocity analyzer (90 degrees curved solenoid, placed downstream the chopper). When the voltage applied to the chopper grid is zero, the positrons come through the chopper grid and are accelerated in the gap between the chopper grid and first drift tube (see Figure 9). Thus, positron pulses with a duration of 300 ns are produced by this way.

In the gap between the drift tube 1 and the buncher tube the velocity of positrons from the $300 \mathrm{~ns}$ pulse is modulated by a nonlinear pulsed voltage applied to the buncher tube relative to the drift tubes. The buncher tube length is determined by a distance-of-flight of positrons entering the buncher during 


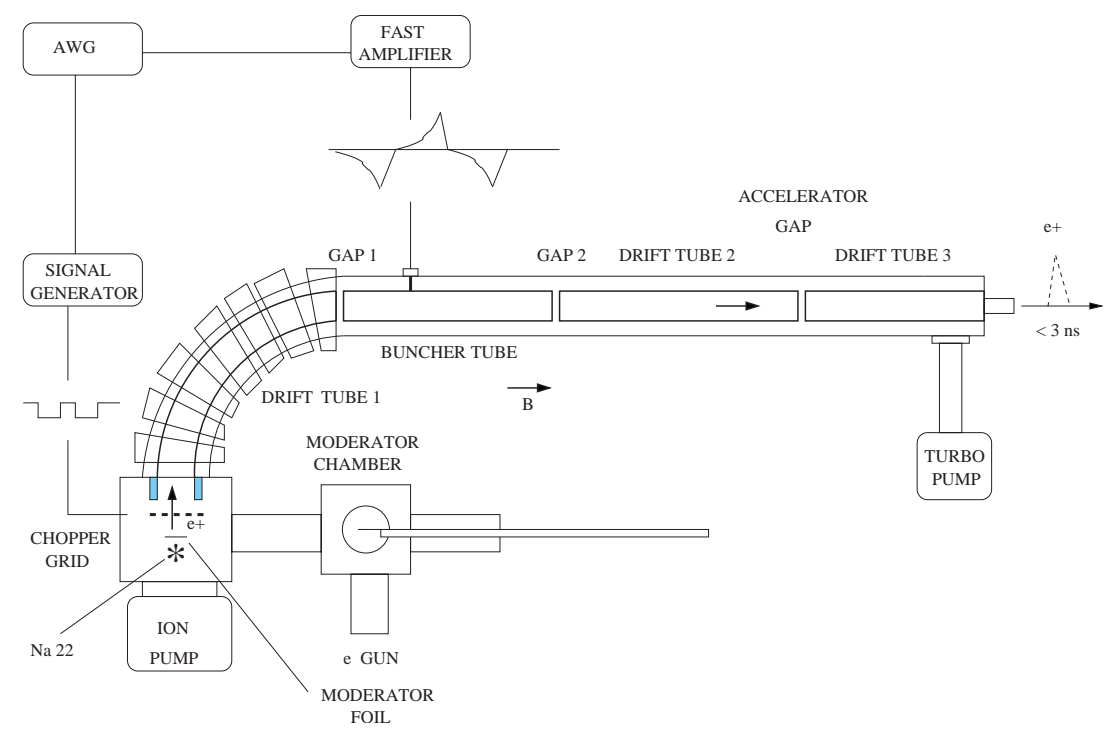

Figure 9: ETHZ-INR experiment: Schematic illustration of the magnetically transported pulsed positron beam.

300 ns. In a second gap between the buncher tube and a drift tube 2 the positron velocity is modulated again by the same voltage pulse applied to the buncher.

The buncher voltage pulse is produced by an arbitrary waveform generator (AWG) connected to a fast post-amplifier whose shape for the two-gap buncher is determined appropriately. In this case is determined by the ratio of the final and initial energy dispersion in the positron beam pulse. Experimentally measured initial energy dispersion of the moderated positrons is about $2 \mathrm{eV}$. Taking into account that the final energy spread in the given two gap buncher is about $200 \mathrm{eV}$ one expects a compression ratio of $\simeq 100$.

The pulsed beam is currently under construction. In 2003 encouraging results in DC mode (See Figure 10) were obtained confirming the correct design of the source, the moderator (see Figure 11) and the magnetic transport system. Assembly of the pulsed beam is foreseen for 2004 .

The experimental signature of the $o-P s \rightarrow$ invisible decay is an excess of events above the background at zero-energy deposition in the ECAL. The $90 \%$-confidence level limit on the branching ratio for the $o-P s \rightarrow$ invisible decay for a background free experiment is given by

$$
S(90 \%)=\frac{N(o-P s \rightarrow \text { invisible })}{N_{o-P s} N_{\text {coll }}}
$$

where $N(o-P s \rightarrow$ invisible $)=2.3$ and the terms in the denominator are the integrated number of produced $o-P s$ 's $\left(N_{o-P s}\right)$, and the average number of $o-P s$ collisions in the cavity, respectively. The number $N_{o-P s}$ is defined as a product $N_{o-P s}=R_{e^{+}} \cdot \epsilon_{o-P s} \cdot \epsilon_{e^{+}} \cdot t$, where the first factor is the number of delivered positrons per second on the target, the second one is the efficiency for $o-P s$ production, and the third one is the efficiency of the secondary electron transportation from the target to the MCP in the positron tagging system. Taking $R_{e^{+}}=2 \times 10^{3} / \mathrm{sec}, \epsilon_{o-P s}=20 \%$ and $\epsilon_{e^{+}}=100 \%$, we expect $\approx 7 \times 10^{7}$ prompt and $\approx 1.7 \times 10^{7} o-P s$ annihilations per day. Thus, $S(90 \%) \simeq 10^{-7}$.

In case of the observation of zero-energy events, one of the approaches would be to measure their number as a function of the residual gas pressure in the cavity. This would allow a good cross-check: relatively small variations of gas pressure results in larger peak variations at zero energy due to the damping of $o-P s \rightarrow o-P s^{\prime}$ oscillations. 


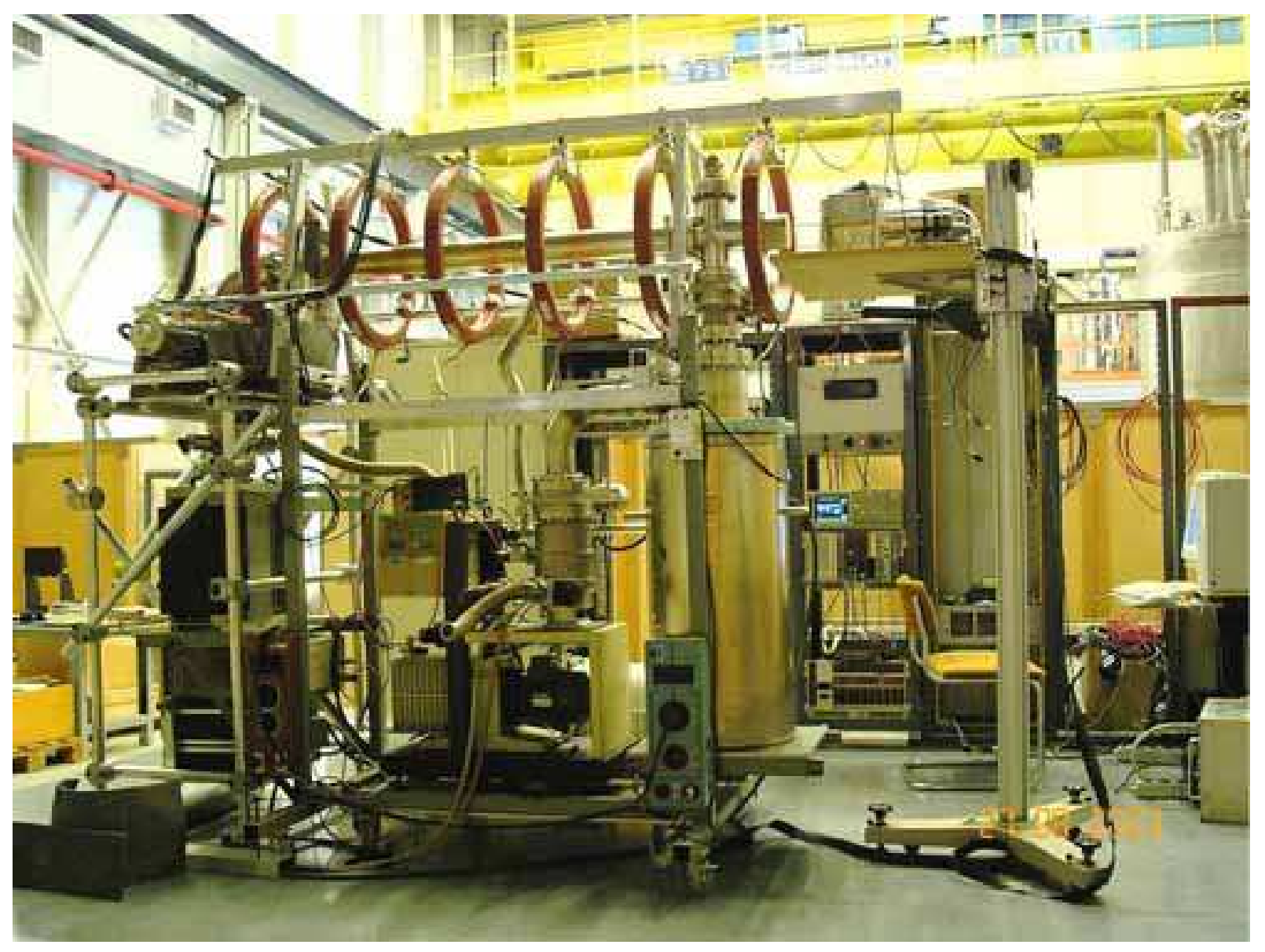

Figure 10: ETHZ-INR setup: Positron beam (DC mode)

\subsection{Berkeley-LLNL experiment}

A collaboration at Lawrence Berkeley and Lawrence Livermore National Laboratories is investigating the feasibility of extending the sensitivity of such an experiment to the level of $10^{-9}$. More information was contributed by Vetter at this workshop 47. One limitation is the counting time required to observe roughly $10^{9} \mathrm{Ps}$ formation triggers, while allowing a several microsecond observation window to allow the o-Ps component to decay away such that the exponential decay probability is less than the desired branching ratio. In such a case, the accidental rate should not give an appreciable probability that a second decay would occur. Elementary calculations suggest that such a detector would require a roughly 3 meter diameter in order to have negligible probability for two $511 \mathrm{keV}$ photons to each leave less than $\approx 100 \mathrm{keV}$ of energy in the detector volume. The total mass of such a volume of liquid scintillator would be roughly $10^{5} \mathrm{~kg}$. Such a geometry naturally addresses the hermeticity requirement, but the sacrifice in compactness and larger mass compared to an array of high-Z inorganic solid scintillators is clear. In such an experiment, the greatest challenge seems to be to provide as clean a trigger as possible for the detector. 


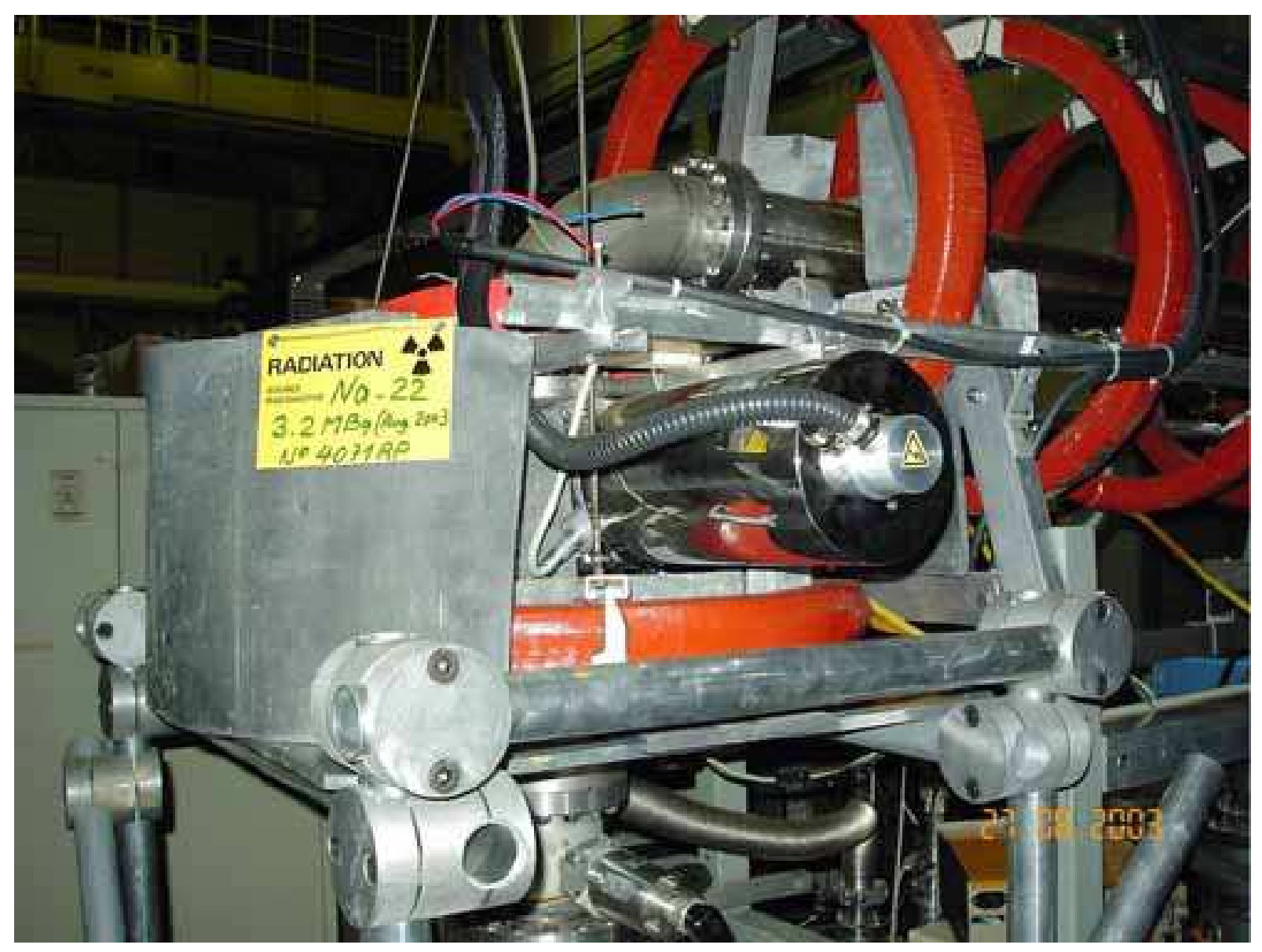

Figure 11: ETHZ-INR setup: Na22 positron source and moderator chamber

\section{Conclusion}

From the large success of this workshop, it is clear that probing fundamental physics with positronium is still an important issue. In this workshop, we have focused on and discussed several new results and addressed new approaches to search for new physics with positronium. Experiments are generally small but difficult and many aspects of the current experiments are not fully understood. Further improved experiments would be valuable information in different domains:

1. in the context of the orthopositronium lifetime, the theoretical precision is now almost two orders of magnitude better than the experimental one. The first priority would be to test with high precision the theoretical prediction of he o-Ps decay rate computed to the second-order correction in $\alpha$. A new generation experiment which could reach a precision at the level of $50 \mathrm{ppm}$ is under investigation.

2. in the context of spectroscopy of positronium, some problems still need to be resolved, like for example the hyperfine splitting of the $1 \mathrm{~S}$ level.

3. In parallel to these precision experiments, new experiments aimed at searching for invisible decays of the orthopositronium are being designed or even constructed, pushing their sensitivity in the range of $10^{-7}$ for the vacuum experiment and $10^{-8}$ for the experiment in aerogel. 
These experiments will allow to further constrain models beyond the standard model. On the other hand, finding such an effect would revolutionize our understanding of the physics of particles.

\section{Acknowledgments}

I wish to express my warmest thanks to all participants of this workshop. The help of collaboration in preparation of this paper and many interesting discussions with P. Crivelli, M. Felcini, S. Gninenko and D. Sillou are greatly appreciated. Support from ETH Zürich, the Swiss National Science Foundation, INR, Moscow and the participation of LMOPS Le Bourget du Lac, LAPP Annecy and CERI Orléans is gratefully acknowledged.

\section{References}

[1] M. Deutsch, Phys. Rev. 82, 455 (1951).

[2] T. D. Lee and C. N. Yang, Phys. Rev. 104 (1956) 254.

[3] L. B. Okun, Int. J. Mod. Phys. A 17S1 (2002) 105 arXiv:hep-ph/0112031.

[4] I. Kobzarev et al., Sov. J. Nucl. Phys. 3, 837 (1966); M. Pavsic, Int. J. Theor. Phys. 9, 229 (1974); R. Foot, H Lew and R. R. Volkas, Phys. Lett. B272, 67 (1991).

[5] Z. Berezhiani, to appear in Proceedings of Workshop on Positronium Physics, Zurich (Switzerland), 30-31 May 2003.

[6] R. Foot, to appear in Proceedings of Workshop on Positronium Physics, Zurich (Switzerland), 30-31 May 2003.

[7] B. Holdom, Phys. Lett. B166, 196 (1986).

[8] R. Foot, A. Yu. Ignatiev and R. R. Volkas, Phys. Lett. B503, 355 (2001) astro-ph/0011156.

[9] S. L. Glashow, Phys. Lett. B167, 35 (1986).

[10] R. Foot and S. N. Gninenko, Phys. Lett. B 480, 171 (2000) arXiv:hep-ph/0003278.

[11] C.I. Westbrook, D.W. Gidley, R.S. Conti and A. Rich, Phys. Rev. A40, 5489 (1989).

[12] J.S. Nico, D.W. Gidley and A. Rich, Phys. Rev. Lett. 65, 1344 (1990).

[13] S. Asai, S. Orito and N. Shinohara, Phys. Lett. B 357, 475-480 (1995).

[14] R. Foot, hep-ph/0308254

[15] R. Bernabei et al. (DAMA Collaboration), Riv. Nuovo Cimento. 26, 1 (2003) astro-ph/0307403 and references there-in.

[16] Z. Berezhiani, D. Comelli and F.L. Villante, Phys. Lett. B503, 362 (2001).

[17] S. L. Dubovsky, V. A. Rubakov and P. G. Tinyakov, Phys. Rev. D 62, 105011 (2000) arXiv:hep-th/0006046.

[18] S. N. Gninenko, N. V. Krasnikov and A. Rubbia, Phys. Rev. D 67, 075012 (2003) arXiv:hep-ph/0302205.

[19] C. I. Westbrook, D. W. Gidley, R. S. Conti, and A. Rich, Phys. Rev. Lett. 581328 (1987). 
[20] O. Jinnouchi, S. Asai and T. Kobayashi, arXiv:hep-ex/0011011

[21] R.S. Vallery, P.W. Zitzewitz and D.W. Gidley, Phys. Lett. 90, (2003).

[22] O. Jinnouchi, S. Asai and T. Kobayashi, Phys. Lett. B 572 (2003) 117 arXiv:hep-ex/0308030.

[23] G. S. Adkins, R. N. Fell, and J. Sapirstein, Phys. Rev. Lett. 845086 (2000) and Ann. Phys. 295136 (2002).

[24] D. Sillou, to appear in Proceedings of Workshop on Positronium Physics, Zurich (Switzerland), 30-31 May 2003.

[25] S. Asai, S. Orito, K. Yoshimura, and T. Haga, Phys. Rev. Lett. 662440 (1991);

S. Orito, K. Yoshimura, T. Haga, M. Minowa, and M. Tsuchiaki, Phys. Rev. Lett. 63597 (1989).

[26] T. Maeno, M. Fujikawa, J. Kataoka, Y. Nishihara, S. Orito, K. Shigekuni, Y. Watanabe, Phys. Lett. B351 574 (1995);

S. Asai, K. Shigekuni, T. Sanuki, and S. Orito, Phys. Lett. B323 90 (1994);

M. Tsuchiaki, S. Orito, T. Yoshida, and M. Minowa, Phys. Lett. B236 81 (1990) 81.

[27] T. Mitsui, R. Fujimoto, Y. Ishisaki, Y. Ueda, Y. Yamazaki, S. Asai, and S. Orito, Phys. Rev. Lett. 702265 (1993).

[28] T. Mitsui, K. Maki, S. Asai, Y. Ishisaki, R. Fujimoto, N. Muramoto, T. Sato, Y. Ueda, Y. Yamazaki and S. Orito, Euro. phys. Lett. 33111 (1996);

A. Badertscher, P. Crivelli, M. Felcini, S.N. Gninenko, N.A. Goloubev, P. Nedelec, J.P. Peigneux, V.Postoev, A. Rubbia and D. Sillou Phys. Lett. B542 29 (2002)

[29] S. Asai, S. Orito, T. Sanuki, M. Yasuda, and T. Yokoi, Phys. Rev. Lett. 661298 (1991);

D. W. Gidley, J. S. Nico, and M. Skalsey, Phys. Rev. Lett. 661302 (1991).

[30] K. Marko and A. Rich, Phys. Rev. Lett. 33980 (1974).

[31] G. S. Atoian, S. N. Gninenko, V. I. Razin and Y. V. Ryabov, Phys. Lett. B 220 (1989) 317.

[32] S. N. Gninenko, Phys. Lett. B 326 (1994) 317.

[33] S.G. Karschenboim, to appear in Proceedings of Workshop on Positronium Physics, Zurich (Switzerland), 30-31 May 2003.

[34] A. Penin, ibid.

[35] C. Smith, ibid.

[36] S. Asai , T. Hyodo, Y. Nagashima, T.B. Chang and S. Orito, Materials Science Forum, 619175 (1995).

[37] S.Asai "New measurement of orthopositronium lifetime", Ph. D. thesis, University of Tokyo (1994); S. Asai, S. Orito, and N. Shinohara, Phys. Lett. B357 475 (1995).

[38] S. Asai, O. Jinnouchi and T. Kobayashi, to appear in Proceedings of Workshop on Positronium Physics, Zurich (Switzerland), 30-31 May 2003.

[39] M. Skalsey, J. J. Engbrecht, R. K. Bithell, R. S. Vallery, and D. W. Gidley, Phys. Rev. Lett. 80, 17 (1998).

[40] D.W.Gidley, J.S. Nico and M. Skalsey, Phys. Rev. Lett. 66, 1302-1305 (1991).

[41] A. P. Mills, Jr. and G. H. Bearman, Phys. Rev. Lett. 34, 246 (1975); A. P. Mills, Jr., Phys. Rev. A27, 262 (1983). 
[42] M. W. Ritter, P. O. Egan, V. W. Hughes, and K. A. Woodle, Phys. Rev. A30, 1331 (1984).

[43] S. N. Gninenko, N. V. Krasnikov and A. Rubbia, Mod. Phys. Lett. A 17, 1713 (2002).

[44] A.Czarnecki and S.G.Karshenboim, hep-ph/9911410 J. Govaerts and M.Van CailliePhys.Lett.B391,451 (1996).

[45] P. Crivelli, to appear in Proceedings of Workshop on Positronium Physics, Zurich (Switzerland), 30-31 May 2003.

[46] S. Gninenko, ibid.

[47] P. Vetter, ibid. 